\title{
Role of Oral Health Directorate-Ministry of Health-Khartoum State-Sudan during COVID-19 Pandemic-May-2020
}

\author{
Gabani W* \\ Ministry of health, Sudan
}

*Corresponding author: Wisam Gabani, Oral Health Directorate, Ministry of Health, Khartoum State, Sudan, Tel: 00249918150778; Email: wisamgabani@hotmail.com

\section{Commentary}

Volume 5 Issue 4

Received Date: October 16, 2020

Published Date: October 27, 2020

DOI: $10.23880 /$ oajds-16000277

\section{Abstract}

The outbreak of COVID19 started from Wuhan, China, last December 2019 and have become a major challenging public health problem not only China but also all the countries around the world. On January 30, 2020, the World Health Organization announced that this outbreak had constituted a public health emergency of international concern. Our traditions in Sudan have contributed a lot to the spread of the virus. The social life of our people is so much connected and there are some practices that they need to drop. The health system in Sudan is suffering a lot of problems makes it difficult to contain the situation easily. The Oral Health Directorate plays a major and leading role in reducing the pain and suffering of the patients complaining from oral and dental problems in respect to the current situation of the pandemic.

Keywords: COVID 19; Dental Management; Oral Health Directorate

Abbreviations: MERS: Middle East Respiratory Syndrome; SARS: Severe Acute Respiratory Syndrome; WHO: World Health Organization; GAVI: Global Alliance for Vaccines and Immunizations; OHD: Oral Health Directorate.

\section{Introduction}

Corona viruses are a large family of viruses that can cause respiratory infections ranging from the common cold to more severe diseases like Middle East Respiratory Syndrome (MERS) and Severe Acute Respiratory Syndrome (SARS). The 2019 novel corona virus is a new strain that has not been identified in humans before. The infectious disease that is caused by it is now called covid-19 [1]. The novel corona virus was initially named $2019-\mathrm{nCoV}$ and officially as severe acute respiratory syndrome corona virus 2 (SARSCoV-2) [1].

A new corona virus COVID-19 was officially announced as the causative pathogen by the Chinese Center for Disease Control and Prevention On January 8, 2020 [2]. The epidemics of COVID19 started from Wuhan, China, last December and have become a major challenging public health problem not only China but also countries around the world [3].

On January 30, 2020, the World Health Organization (WHO) announced that this outbreak had constituted a public health emergency of international concern [1]. As of February 26, COVID-19 has been recognized in 34 countries, with a total of 80,239 laboratory-confirmed cases and 2,700 deaths [1]. The common transmission routes is by respiratory droplets (droplet inhalation transmission) expelled through the nose or mouth when the individual coughs or sneezes; and contact transmission (with oral, nasal, and eye mucous membranes) [4]. It can also be transmitted indirectly through the contact of the hands or mucous membranes with the contaminated inert surface. Reports differ on the number of days that the virus can survive on those surfaces. In addition, studies have shown that it can be transmitted directly or indirectly through saliva [4]. Also studies suggested that COVID 19 may be airborne through aerosols formed during medical procedures [4].

Dental care settings carry the risk of COVID 19 infection due to the specificity of its procedures, which involves faceto-face communication with patients, and frequent exposure 


\section{Open Access Journal of Dental Sciences}

to saliva, blood, and other body fluids, and the handling of sharp instruments. The pathogenic microorganisms can be transmitted in dental settings through inhalation of airborne microorganisms that can remain suspended in the air for long periods, direct contact with blood, oral fluids, or other patient materials, contact of conjunctival, nasal, or oral mucosa with droplets and aerosols containing microorganisms generated from an infected individual and propelled a short distance by coughing and talking without a mask, and indirect contact with contaminated instruments and/or environmental surfaces 8 . In addition to blood and salivary contamination, the majority of routine dental treatments generate significant amounts of droplets and aerosols due to the utilization of devices and equipment such as ultrasonic scalers, air-water syringes and air turbine hand pieces [5].

Given the high transmissibility of the disease; and considering that routine dental procedures usually generate aerosols; during the course of this pandemic, alterations to dental treatment should be considered to maintain a healthy environment for the patients and the dental team [5]. Also in order to preserve personal protective equipment and patient care supplies and expand available hospital capacity during the COVID-19 pandemic, the Centers for Disease Control and Prevention (CDC) recommends that dental facilities postpone elective procedures, surgeries, and non-urgent dental visits, and prioritize urgent and emergency visits and procedures now and for the coming several weeks [6].

\section{Situation of COVID 19 in Sudan}

Our traditions in Sudan have contributed a lot to the spread of the virus. The social life of our people is so much connected and there are some practices that they need to drop [7]. After a steep jump in confirmed COVID-19 cases, Sudan's transitional government has imposed a nationwide lockdown to try to stop the spread of virus. The government banned all travel from Khartoum to other parts of the country and vice versa [7].

Up-to-date the Sudan Federal Ministry of Health declared that till today 4th of May COVID 19 spread out in 15 states in Sudan. The total number of confirmed cases is 678, out of which, 576 active cases, 61 are totally recovered and 41 died. Most of the cases (594) are in Khartoum state. By this statistics Sudan is considered as one of the countries that have high mortality rate due to COVID 19.-9.2\% [8].

\section{Challenges Facing the Health System in Sudan to Contain COVID 19 Pandemic}

On January 30, 2020, the International Health Regulations Emergency Committee of the World Health Organization declared the 2019-nCoV outbreak a "Public
Health Emergency of International Concern (PHEIC). This decision was mainly to preventspread of the virus in countries with weakest health systems so that all countries could be prepared for active surveillance, early detection, isolation and case management, contact tracing and mitigation of onward spread of COVID-19 infection [9].

The health system in Sudan is marked by decades of limited to no investment, underfunding and lack of qualified staff, infrastructure, equipment, medicines and supplies. The surveillance system doesn't cover the entire country and is structurally weak with long delays between alert and confirmation of an outbreak. Sudan lacks sufficient and adequately trained medical staff to support increased demand, isolation units, intensive care units, infection control materials, medicines and medical supplies to address quickly spreading outbreaks including the corona virus (covid-19) in all states across the country. COVID-19 cases may force health facilities to close to other patients due to isolation procedures. Regular treatments for malnutrition or maternal care may have to be suspended [9].

Different international organizations such as the WHO, UNDP and the UNICEF support the Federal Ministry of Health to elaborate the countrywide preparedness in term of strategies and plans, guidelines and protocols, building capacities, provision of the infection prevention and control supplies, mobilization of additional resources from the Global Fund and Global Alliance for Vaccines and Immunizations (GAVI) [9].

\section{Role of Oral Health Directorate}

The Oral Health Directorate (OHD)-Ministry of Health Khartoum State is a one of the Curative Medicine sectors. It is responsible for all public dental clinics in Khartoum state. OHD has 163 dental clinics spread over the seven localities in Khartoum state. The majority of the clinics found within a primary health centre as part of the primary health care, others are located within some hospitals in Khartoum state [10].

The OHD plays a major and leading role in reducing the pain and suffering of the patients complaining from oral and dental problems in respect to the current situation of the pandemic. According to the $\mathrm{CDC}$ and $\mathrm{WHO}$ recommendations OHD stated that: all public dental clinics in Khartoum state should seized the elective dental procedures and restrict their activities to the emergency treatment. Then a decision was taken to close all the public dental clinics except that in the hospitals and other selective dental clinics in the centre of the city so as to be nearby most of the citizens [10].

It collaborates with the Federal Ministry of Health 


\section{Open Access Journal of Dental Sciences}

and develops a guideline on the light of that elaborated by the WHO and the CDC for dental practitioners [10]. This guideline gives the dentists broad outlines for whom, what and when to manage patients come to the dental clinics so as to prevent both the dentists and the patients and to preserve the limited Personal Protective Equipment resources with respect to all infection prevention and control measures [11]. The emergency dental conditions include: continuous pain that cannot be relieved by analgesics, swelling specially that interfere with breathing and trauma [11].

Also the guideline directs the dentists to implement the visual triage check list developed by Sudan Federal Ministry of Health before examining the patients. Protocol was developed outlining actions should be taken to well deal with suspicious cases [11]. In addition, the guideline encourages the dentists to raise patients' awareness about the corona viruses, its mode of transmission and ways of prevention, so as to act actively in educating the patients about the disease [11].

Latter on; and as the situation get worsen in Khartoum state, only four dental facilities are prepared to receive patients with emergency dental situations from 8 a.m to 8 p.m, during the whole week in two shifts, morning and evening [10]; which are Khartoum Dental Teaching Hospital, Academy Teaching Hospital, Dental Department In Omdurman Hospital and Haj Alsafi Dental Centre [10].

Regarding the dental staff working in the public sector, they listed their names and distributed to the nearest working dental facility to provide the necessary procedures needed for emergency cases, as the oral health directorate make transportation and a meal available for them. The staff was well trained on how to implement the triage, how to deal with suspicious cases and how to follow the needed infection control measures. All this was arranged by the coordination between the oral health services department in the OHD, the Dental Directors in the localities and the dentists [10].

Furthermore the OHD provide sustainable supply for the working clinics with all Personal Protective Equipments that needed by the dentists to manage the patients in addition to other disinfection materials like hand wash soap and sanitizers. OHD restricts the use of all PPEs: N95 mask, face shield, disposable aprons, head and foot cover [10].

OHD create a web site to provide a comprehensive overview and up to date information on the pandemic alerts and response information each day, proper personal protection precautions, workers and patients' safety, and other concerns allowing the dental professionals to keep current on important issues in protection to help keep themselves and their patients safe [10].
Another initiative was done by the Oral Health Directorate and Sudanese Dental Specialists Assembly in coordination with a communication companies; they open a call centre services to provide a consultations and reply to the patients enquires in all specialties regarding oral and dental problems through a unified phone number. Thirty dental specialists have got mobile phones to reply the patients' complains at their homes without taking the risk of going out and exposed to the virus [10].

\section{References}

1. Meng L, Hua F, Bian Z (2020) Coronavirus disease 2019 (COVID-19): emerging and future challenges for dental and oral medicine. Journal of Dental Research 99(5): 481-487.

2. He F, Deng Y, Li W (2020) Coronavirus Disease 2019 (COVID-19): What we know? Journal of Medical Virology 92(7): 719-725.

3. Wu Z, McGoogan JM (2020) Characteristics of and important lessons from the coronavirus disease 2019 (COVID-19) outbreak in China: summary of a report of 72314 cases from the Chinese Center for Disease Control and Prevention. Jama 323(13): 1239-1242.

4. Peng X, Xu X, Li Y, Cheng L, Zhou X, et al. (2020) Transmission routes of $2019-\mathrm{nCoV}$ and controls in dental practice. International Journal of Oral Science 12(1): 1-6.

5. Alharbi A, Alharbi S, Alqaidi S (2020) Guidelines for dental care provision during the COVID-19 pandemic. The Saudi Dental Journal.

6. (2020) CDC Guidelines for Dental Treatment on COVID 19.

7. https://www.voanews.com/covid-19-pandemic/jumpsudan-coronavirus-cases-prompts-total-lockdown.

8. Corona virus disease (COVID-19) Situation Report-105 Data as received by WHO from national authorities by 10:00 CEST, 4 May 2020.

9. Sudan Corona Virus COVID 19-Country Preparedness and Response Plan CPRP.

10. Oral Health Directorate, COVID 19 Emergency Plan / 2020. (Unpublished Document).

11. Guidelines for dental professionals working in the Public Sector on Dental Management during the COVID 19 period. 